\title{
Undervaccination of Perinatally HIV-Infected and HIV-Exposed Uninfected Children in Latin America and the Caribbean
}

\author{
Regina C. M. Succi, MD1, Margot R. Krauss, MD², D. Robert Harris, PhD², Daisy M. \\ Machado, MD ${ }^{1}$, Maria Isabel de Moraes-Pinto, MD ${ }^{1}$, Marisa M. Mussi-Pinhata, MD ${ }^{3}$, Noris \\ Pavia Ruz, MD ${ }^{4}$, Russell B. Pierre, MD $^{5}$, Lenka Kolevic, MD $^{6}$, Esau Joao, MD ${ }^{7}$, Irene \\ Foradori, MD $^{8}$, Rohan Hazra, MD $^{9}$, George K. Siberry, MD $^{9}$, and for the NISDI Pediatric \\ Study Group 2012* \\ ${ }^{1}$ Escola Paulista de Medicina, Universidade Federal de São Paulo, UNIFESP, São Paulo, Brasil \\ ${ }^{2}$ Westat, Rockville, MD, USA \\ ${ }^{3}$ Faculty of Medicine of Ribeirão Preto, University of São Paulo, Ribeirão Preto, Brazil \\ ${ }^{4}$ Clínica de Inmunodeficiencias/Depto. de Infectología, Hospital Infantil de Mexico Federico \\ Gomez, Mexico City \\ ${ }^{5}$ Department of Child \& Adolescent Health, the University of the West Indies, Jamaica \\ ${ }^{6}$ National Institute of Child Health and Faculty of Medicine of National University of San Marcos of \\ Lima Peru \\ ${ }^{7}$ Hospital Federal dos Servidores do Estado, Rio de Janeiro, Brasil \\ ${ }^{8}$ Facultad de Medicina, Universidad de Buenos Aires (UBA), Ciudad de Buenos Aires, Argentina \\ ${ }^{9}$ Pediatric Adolescent Maternal AIDS Branch, Eunice Kennedy Shriver National Institute of Child \\ Health and Human Development, National Institutes of Health, Bethesda, MD, USA
}

\begin{abstract}
Background-Perinatally HIV-infected children (PHIV) may be at risk of undervaccination. Vaccination coverage rates among PHIV and HIV-exposed uninfected children (HEU) in Latin America and the Caribbean were compared.

Methods-All PHIV and HEU children born from 2002-2007 that were enrolled in a multi-site observational study conducted in Latin America and the Caribbean were included in this analysis. Children were classified as up to date (UTD) if they had received the recommended number of doses of each vaccine at the appropriate intervals by 12 and 24 months of age. Fisher's exact test was used to analyze the data. Covariates potentially associated with a child's HIV status were considered in multivariable logistic regression modeling.
\end{abstract}

\footnotetext{
Correspondence to: George K. Siberry.

Corresponding Author: George K Siberry, MD, MPH, Medical Officer, Pediatric, Adolescent, and Maternal AIDS (PAMA) Branch, Eunice Kennedy Shriver National Institute of Child Health and Human Development, National Institutes of Health, 6100 Executive Boulevard, Room 4B11H, Bethesda, MD 20892-7510, (P) 301.496.7350, (F): 301.496.8678, siberryg@ mail.nih.gov.

Publisher's Disclaimer: This is a PDF file of an unedited manuscript that has been accepted for publication. As a service to our customers we are providing this early version of the manuscript. The manuscript will undergo copyediting, typesetting, and review of the resulting proof before it is published in its final citable form. Please note that during the production process errors may be discovered which could affect the content, and all legal disclaimers that apply to the journal pertain.

Conflict of Interest: All authors report that they do not have an association that might pose a conflict of interest.
} 
Results-Of 1156 eligible children, 768 (66.4\%) were HEU and 388 (33.6\%) were PHIV. HEU children were significantly $(\mathrm{p}<0.01)$ more likely to be UTD by 12 and 24 months of age for all vaccines examined. Statistically significant differences persisted when the analyses were limited to children enrolled prior to 12 months of age. Controlling for birth weight, sex, primary caregiver education and any use of tobacco, alcohol or illegal drugs during pregnancy did not contribute significantly to the logistic regression models.

Conclusions-PHIV children were significantly less likely than HEU children to be UTD for their childhood vaccinations at 12 and 24 months of age, even when limited to children enrolled before 12 months of age. Strategies to increase vaccination rates in PHIV are needed.

\section{Keywords}

Pediatric HIV infection; vaccination; Latin America

Preventive health care, including immunization, is an essential part of comprehensive care that can minimize adverse health outcomes for perinatally HIV-infected children and adolescents. Based on evidence of safety, immunogenicity and, in some cases, efficacy of most childhood vaccines for children with HIV infection, guidelines [1, 2, 3] have been developed for recommended immunization in this population. However, health care staff responsible for immunization delivery may miss opportunities for immunizing HIV-infected children because they are unaware of those recommendations, they are concerned about greater risk with use of vaccines in this population, or other reasons. African $[4,5]$ and European studies $[6,7,8]$ found lower immunization coverage of HIV-infected children compared to uninfected peers, while a North American study found equally low vaccine coverage, for both HIV-infected and the HIV-exposed uninfected populations for some vaccines [9]. In a rural South African population, maternal HIV-positive status was independently associated with lower vaccination rates for four vaccines in children 12-23 month of age (BCG, DTP3, poliomyelitis, and hepatitis B) [10].

Childhood immunization adequacy can be used as an indicator of children's primary health care and perhaps highlight areas for improvement. Yet, immunization coverage for HIVinfected children in Latin America and the Caribbean has not been described. Given the importance of vaccination for HIV-infected children and the data from other regions suggesting that they are at higher risk of under-immunization, we evaluated immunization rates, vaccine adverse events (cases associated with polio and BCG vaccines), and the occurrence of vaccine preventable diseases among HIV-exposed uninfected (HEU) and perinatally HIV-infected (PHIV) children in Latin America enrolled in the Eunice Kennedy Shriver National Institute of Child Health and Human Development (NICHD) International Site Development Initiative (NISDI).

\section{METHODS}

The NISDI Pediatric protocol was a prospective cohort study that followed HIV-infected and perinatally HIV-exposed, uninfected children at multiple clinical sites in Latin America and the Caribbean. A description of an early version of the protocol cohort has been published [11]. When enrollment began in October 2002, HEU infants (up to 12 months of age) and HIV-infected children ( $\leq 21$ years of age) were enrolled from eleven participating sites in Brazil and two sites each in Mexico and Argentina; in 2006 sites in Peru and Jamaica were added. The next version of the protocol, initiated in 2008, continued follow-up of HIVinfected children previously enrolled by six years of age and also enrolled a new group of HIV-infected children less than six years of age, at twelve Brazilian sites and one original site each in Mexico and Peru. The protocol was approved by the ethical review boards of each participating clinical site, by the sponsoring institution (NICHD), the data management 
and statistical center (Westat), the Peruvian Ministry of Health and the Brazilian National Ethics Committee (CONEP). Informed consent was obtained from parents or guardians of minor participants.

Eligibility for this analysis was limited to HEU and PHIV children born from 2002 through 2007 to control for potential variation in vaccination practice. HEU children were enrolled up to one year of age, while PHIV children were enrolled up to their sixth birthday. All children were born to women with documented HIV infection. Those classified as PHIV had no risk factors other than their mother's HIV infection and were confirmed HIV-infected on the basis of positive HIV tests on two different occasions (virologic tests if under age 18 months, virologic and/or antibody tests if age $\geq 18$ months). HEU children had negative HIV virologic testing at ages $\geq$ one month and $\geq$ four months or repeatedly negative HIV antibody testing at age $\geq 6$ months. Maternal information was collected on all enrolled children, including the reason for taking antiretroviral (ARV) drugs (prophylaxis or treatment), educational level of the primary care provider and substance use during pregnancy. All enrolled children had the following information collected: gestational age, birth weight, and vaccinations received from birth up to the age of enrollment. All children were medically evaluated in a standardized fashion every six months, including laboratory assessments (CD4+ T-lymphocyte [CD4] \%, CD4 counts and HIV viral load, if appropriate), and additional vaccinations.

Children were considered up to date (UTD) for a specific vaccine if they had received the recommended number of doses for that vaccine, and the doses followed minimum age and minimum interval guidelines according to World Health Organization recommendations [12]. The definitions for UTD at 12 months by vaccine were: one dose of Bacille CalmetteGuérin vaccine (BCG); three doses of oral polio vaccine (OPV) or two doses of inactivated poliovirus vaccine (IPV); three doses of diphtheria-tetanus-pertussis-containing vaccines (DTP); three doses of Haemophilus influenzae type b vaccine (Hib); and three doses of hepatitis B vaccine (HBV). A child was considered UTD for all vaccines at 12 months if they had received all recommended doses for BCG, OPV/IPV, DTP, Hib, and HBV at the appropriate dosing intervals.

A child was considered to be UTD at 24 months of age for each vaccine as follows: three doses of either OPV or IPV; four doses of DTP; three doses of Hib prior to 12 months of age or at least one dose of Hib given after 12 months of age; three doses of HBV; and one dose of measles-containing vaccine (MV). A child was considered UTD for all vaccines at 24 months of age if they had received all recommended doses for OPV/IPV, DTP, Hib, HBV, and MV at the appropriate intervals.

Vaccine related events (vaccine-associated paralytic polio and adverse BCG vaccine events) and vaccine-preventable diseases (measles, hepatitis B, Hib, pertussis, and polio) were ascertained at the twice yearly study visits.

\section{Statistical Methods}

Fisher's exact test was used to evaluate the relationship of demographic characteristics with HIV status. Children were also classified according to vaccine status (UTD, Not UTD, Insufficient follow-up) for each vaccine at 12 and 24 months of age and Fisher's exact test was used to assess its relationship with HIV infection status.

To investigate potential for bias related to younger age at study enrollment of HEU children compared to PHIV children, the analyses were repeated including just the subset of PHIV children enrolled before 12 months of age who were still on study at 24 months of age. 
Covariates associated with a child's HIV status ( $\mathrm{p} \unlhd$ ().2) were considered candidates for inclusion in multivariable logistic regression modeling. All analyses were conducted using SAS statistical software, version 9.2 (SAS Institute Inc. Cary, NC).

\section{RESULTS}

Among the 1926 children enrolled in the NISDI Pediatric protocol, 1161 were born between 2002 and 2007. Five HIV infected children who were horizontally infected were excluded from the analysis. Of the remaining 1156, 768 (66.4\%) were HEU and 388 (33.6\%) were PHIV. Most participants $(843,72.9 \%)$ were enrolled in Brazil. Variables found to be significantly associated with children's HIV status included: country of birth; education of the primary caregiver; reason for maternal ARV use; ever use of tobacco and alcohol and illegal drugs during pregnancy; and child's age at enrollment into NISDI (Table 1).

HEU children were significantly $(\mathrm{p}<0.01)$ more likely to be UTD by 12 months of age for each vaccine; depending on the specific vaccine examined, the probability of being UTD for HEU was approximately two to seven times that of being UTD if PHIV (Table 2).

Differences in UTD status between HEU and PHIV were large even for non-live vaccines: Hib (73.3\% vs. $52.7 \%)$, HBV ( $85.9 \%$ vs. $61.8 \%)$ and DTP (79.7\% vs. $60.5 \%)$. At 24 months of age, HEU children remained significantly $(\mathrm{p}<0.01)$ more likely to be UTD for each vaccine, including measles vaccine; the probability of being UTD for HEU was approximately two to three times that of PHIV (Table 2). These differences persisted when the analyses were limited to PHIV children enrolled prior to 12 months of age on the NISDI study (Table 3).

Since most children were from Brazil, the results were driven largely by what occurred among Brazilian study participants. However, sub-analyses of vaccination status by country demonstrated that Peru experienced the same statistically significant differences by HIV status for all vaccines while Argentina showed the same pattern, although the differences reached statistical significance only for hepatitis B (data not shown). There were too few HEU children in Mexico and Jamaica (one and three HEU children, respectively) for any meaningful analyses.

HEU children had a greater probability (odds ratio $=2.4 ; 95 \%$ CI: $1.8,3.0$ ) of being UTD on all vaccines at 12 months of age than PHIV, with 57.4\% of HEU and 36.9\% of PHIV having received all recommended doses for BCG, Hib, HBV, DTP and polio by their first birthday (Table 4). The probability of being fully immunized by their second birthday for HEU continued to be two times that of PHIV (95\% CI: 1.6-2.6); $48.8 \%$ of HEU and $31.8 \%$ of PHIV received all the recommended doses for Hib, HBV, DPT, Polio and MV.

A series of logistic regression models were fit to the data for the dependent variables: BCG UTD by 12 months of age, MV UTD at 24 months of age and HBV UTD by 24 months of age, controlling for primary caregiver education, any use of tobacco, alcohol, marijuana or cocaine during pregnancy, and child's birth weight and gender. None of these covariates contributed significantly to the models, and each resulted in only modest changes in the association of HIV status with vaccine UTD status. Site, age at enrollment and maternal reason for ARV were not included in the final modeling as their distributions would otherwise lead to model failure.

No cases of vaccine-associated paralytic polio were reported. However, there were fourteen cases of adverse events related to BCG vaccination, all reported among PHIV children: Among BCG vaccinated PHIV children, 4.8\% (95\% CI: 2.7-8.1\%) experienced a regional 
event (13 of 273 vaccinated PHIV children) and $0.4 \%$ (95\% CI: 0-2.2\%) had a systemic BCG related event (1 of 273 vaccinated PHIV children).

Both cases of vaccine preventable diseases occurred in PHIV children and were confirmed by culture. : one case of measles in a 20-month-old Brazilian boy who had received one dose of MMR at 13 months of age and one case of Hib pneumonia in a 22-month-old Brazilian boy who had received three doses of Hib prior to one year of age. The child diagnosed with measles in 2006 had been receiving didanosine and zidovudine for five months and had a viral load of 2,000,000 copies/mL; no CD4\% was available prior to or at the time of diagnosis. The case of Hib pneumonia occurred in 2009 in a child who had been receiving zidovudine, abacavir and lamivudine for nearly two years, and had a CD4 percent of $24 \%$ and viral load of 9,867 copies/mL two months prior to the Hib infection; his CD4\% nadir prior to the episode was $15 \%$.

\section{DISCUSSION}

The results of this study indicate that PHIV and HEU children in Latin America are at high risk of being incompletely immunized at 12 and 24 months of age. The proportions of children UTD for routine vaccines at 12 months of age ranged from $52.7 \%$ for Hib vaccine (PHIV group) to $94.2 \%$ for BCG (HEU group). HEU children had significantly better coverage than PHIV children for all vaccines examined. HEU children did experience vaccine coverage under $80 \%$ for Hib, DTP, and polio at 12 months of age; however, by 24 months of age vaccine coverage was greater than $80 \%$ for all vaccines except the fourth dose of DTT. Although there was an increase in vaccination among PHIV children from 12 to 24 months of age, they had less than $75 \%$ coverage for each vaccine. The decline in percentage coverage for DTP from 12 to 24 months of age for both PHIV and HEU children (60.5\% to $43.5 \%$ and $79.7 \%$ to $74.2 \%$, respectively) demonstrates that the recommended fourth dose of DTP was not routinely administered. Measles vaccination was completed for $68.1 \%$ of the PHIV and $89.9 \%$ of the HEU children by 24 months of age.

Since HEU children were required to enroll prior to age 12 months and PHIV children were enrolled up to age 6 years, we hypothesized that the difference found between these two groups in vaccination status might be attributable to the fact that children enrolled in NISDI prior to their first birthday were being monitored more closely and thus more likely to receive vaccinations than children enrolled after 12 months of age. In addition, children enrolled at this earlier age may have had fuller ascertainment of the vaccine administered in the first year of life. However, even when the analyses were restricted to subjects enrolled in NISDI prior to their first birthday (Table 3), immunization coverage remained significantly poorer for PHIV children.

As with any observational study, we recognize that the data may be incomplete, such as the reporting of vaccine administrations and the occurrence of minor vaccine related adverse events. However, by restricting our study population to the same birth cohort and using standard definitions to define vaccine doses for determining up to date status, we believe that systematic biases have been minimized and do not account for our findings.

In a study of more than 17,700 Brazilian children born in $2005,81 \%$ had received three doses each of OPV, DTP+Hib, hepatitis B, and one dose each of BCG and MMR vaccines by age 18 months [13]. However, the coverage rate in that study dropped to $68 \%$ when only valid doses were counted. When categorizing 3 doses of DTP as complete as in this Brazilian study, the coverage rate in our study for all vaccines in HEU children at 24 months exceeded 80\% (data not shown). For individual vaccines, the proportion in the Brazilian study who received the full number of valid doses exceeded $90 \%$ for BCG, DTP (3 doses), 
and polio and was between 80 and $90 \%$ for Hib, Hepatitis B and measles. Thus, the coverage rates observed in HEU children in our study were similar to the national vaccine coverage rates for children in Brazil, suggesting good ascertainment of vaccination history in our study. .

Good virologic and immunologic response after 12 months of ART among HIV-infected children in resource-limited settings are comparable to those observed among children in developed countries [14]. The success in treatment of HIV infection in children has resulted in more perinatally HIV-infected children growing up with HIV infection, even in low- and middle-resource countries. For this group of children, the vaccination issue has increasing importance, as HIV infection and associated diseases place them at risk for severe vaccinepreventable diseases and their adverse consequences.

Concern about adverse events may contribute to underimmunization of PHIV children. However, it is known that non-live vaccines are not associated with increased risks of complications in HIV-infected children; in contrast, replicating live vaccines could pose risks for these children. Regional and extra regional localized and disseminated diseases have been reported in association with $B C G$ vaccine $[15,16,17]$. The rate of $B C G$ adverse events in this study [4.8\% (95\% CI: $2.7-8.1 \%$ ) for regional events and $0.4 \%$ (95\% CI: 0 $2.2 \%$ ) for distant BCG related events] was consistent with a Brazilian report which found three cases $(4.5 \%)$ of regional BCG disease and no cases of distant events among $66 \mathrm{HIV}$ infected children [18].

The risk of vaccine-associated paralytic poliomyelitis after OPV is theoretically increased in HIV-infected individuals, but only two cases of vaccine-associated paralytic poliomyelitis have ever been reported in HIV-infected children [19, 20]. No cases were observed in our study and though it is possible that a case occurred but was not captured, this clinical syndrome is unlikely to have been missed with prospective study visits every 6 months. As suggested by Moss et al, the benefits of poliovirus vaccination far outweigh the potential risks in HIV-infected children [15].

Fewer adverse events due to live attenuated vaccines and better response to vaccination, with fewer cases of vaccine preventable diseases, could be attained if children were immunized when their immune systems are not suppressed [21, 22].

The traditional risk factors for inadequate immunization coverage such as poverty, mothers with low level of education and remote access to the health care service were present in this population; however, this did not explain the difference in vaccination rates between HEU and PHIV children.

Given the recognized impact of these vaccines on reducing childhood morbidity and mortality, the failure in vaccination coverage in the PHIV population is disappointing. Two factors might help explain these results: 1) missed opportunities to complete routine scheduled vaccinations because the children had symptomatic HIV infection or were hospitalized; and 2) misconception and myths about immunization of HIV-infected children among their parents and even physicians' apprehension related to vaccination.

Although vaccination is an efficacious preventive measure, as well as an economic rational choice [23], this study highlights poor compliance with routine vaccination of PHIV children in Latin America. Failing to maximize protection in these children against vaccinepreventable diseases not only puts them at risk of substantial morbidity, but also increases the risk of transmission of vaccine-preventable infections to other susceptible members of their communities. New strategies to increase adequate immunization compliance and better protect children from vaccine preventable diseases in this population appear warranted. 


\section{Acknowledgments}

We thank the children and families who participated in the NISDI pediatric project and the site staff involved in the conduct of the study.

Supported by: NICHD Contract \# N01-HD-3-3345 (2002-2007) and by NICHD Contract \# HHSN267200800001C (NICHD Control \#: N01-HD-8-0001) (2007-2012).

\section{*NISDI Pediatric Study Group 2012}

Principal investigators, co-principal investigators, study coordinators, data management center representatives and NICHD staff include: Brazil: Belo Horizonte: Jorge Pinto, Flávia Faleiro (Universidade Federal de Minas Gerais); Caxias do Sul: Rosa Dea Sperhacke, Nicole Golin, Sílvia Mariani Costamilan (Universidade de Caxias do Sul/Serviço Municipal de Infectologia); Nova Iguacu: Jose Pilotto, Beatriz Grinsztejn, Valdilea Veloso, Luis Felipe Moreira, Ivete Gomes (Hospital Geral Nova de Iguacu - HIV Family Care Clinic); Porto Alegre: Rosa Dea Sperhacke, Breno Riegel Santos, Rita de Cassia Alves Lira (Universidade de Caxias do Sul/Hospital Conceição); Rosa Dea Sperhacke, Mario Ferreira Peixoto, Elizabete Teles (Universidade de Caxias do Sul/Hospital Fêmina); Rosa Dea Sperhacke, Marcelo Goldani, Carmem Lúcia Oliveira da Silva, Margery Bohrer Zanetello (Universidade de Caxias do Sul /Hospital de Clínicas de Porto Alegre); Regis Kreitchmann, Marcelo Comerlato Scotta, Debora Fernandes Coelho (Irmandade da Santa Casa de Misericordia de Porto Alegre); Ribeirão Preto: Marisa M. Mussi-Pinhata, Maria Célia Cervi, Márcia L. Isaac, Fernanda Tomé Sturzbecher, Bento V. Moura Negrini (Hospital das Clínicas da Faculdade de Medicina de Ribeirão Preto da Universidade de São Paulo); Rio de Janeiro: Ricardo Hugo S. Oliveira, Maria C. Chermont Sapia (Instituto de Puericultura e Pediatria Martagão Gesteira); Esau Custodio Joao, Maria Leticia Cruz, Ana Paula Antunes, Jacqueline Anita de Menezes (Hospital dos Servidores do Estado); São Paulo: Regina Celia de Menezes Succi, Daisy Maria Machado (Escola Paulista de Medicina- Universidade Federal de São Paulo); Marinella Della Negra, Wladimir Queiroz, Yu Ching Lian (Instituto de Infectologia Emilio Ribas); Mexico: Mexico City: Noris Pavía-Ruz, Dulce MoralesPérez, Jorge Gamboa-Cardeña (Hospital Infantil de México Federico Gómez); Peru: Lima: Jorge Alarcón Villaverde (Instituto de Medicina Tropical "Daniel Alcides Carrión"- Sección de Epidemiologia, UNMSM), María Castillo Díaz (Instituto Nacional de Salud del Niño), Mary Felissa Reyes Vega (Instituto de Medicina Tropical "Daniel Alcides Carrión" Sección de Epidemiologia, UNMSM); Data Management and Statistical Center: Yolanda Bertucci, Laura Freimanis Hance, René Gonin, D. Robert Harris, Roslyn Hennessey, Margot Krauss, James Korelitz, Kathryn Miller, Sharon Sothern de Sanchez, Sonia K. Stoszek (Westat, Rockville, MD, USA); NICHD: Rohan Hazra, Lynne M. Mofenson, George K. Siberry (Eunice Kennedy Shriver National Institute of Child Health and Human Development, Bethesda, Maryland).

The findings and conclusions in this report are those of the authors and do not necessarily represent the views of the National Institutes of Health or the Department of Health and Human Services.

\section{REFERENCES}

1. Menson E, Mellado M, Bamford A, et al. the Paediatric European Network for Treatment of AIDS (PENTA) Vaccines Group, PENTA Steering Committee and Children's HIV Association (CHIVA). Guidance on vaccination of HIV-infected children in Europe. HIV Med. 2012; 13(6):333-336. [PubMed: 22296225] 
2. Ministry of Health, Brazil. Supplement I immunizations. Brazilia, DF: 2010. Antiretroviral Therapy recomendations for children and adolescents infected with HIV. Available at: http:// www.aids.df.gov.br/sites/400/446/00000062.pdf

3. Mofenson LM, Brady MT, Danners SP, et al. Guidelines for the Prevention and Treatment of Opportunistic Infections Among HIV-Exposed and HIV-Infected Children: Recommendations from CDC, the National Institutes of Health, the HIV Medicine Association of the Infectious Diseases Society of America, the Pediatric Infectious Diseases Society, and the American Academy of Pediatrics. MMWR Recommendations and Reports. 2009 Sep 4.Vol. 58(No. RR-11)

4. Mast TC, Kigozi G, Wabwire-Mangen F, Sewankambo N, et al. Immunization coverage among children born to HIV-infected women in Rakai district, Uganda: effect of voluntary testing and counseling (VCT). AIDS Car. 2006; 18(7):755-763.

5. Setse RW, Cutts F, Monze M, et al. HIV-1 Infection as a risk factor for incomplete childhood immunization in Zambia. J Trop Pediatr. 2006; 52:324-328. [PubMed: 16401614]

6. Fernandez-Ibieta M, Ramos-Amador JT, Aunon-Martin I. HIV-infected children vaccination coverage and safety in a Western European cohort: a retrospective study. Int J STD AIDS. 2007; 18:351-353. [PubMed: 17524201]

7. Myers C, Posfay-Barbe KM, Aebi C, et al. the Pediatric Infectious Disease Group of Switzerland (PIGS), and the Swiss Mother and Child HIV Cohort Study (MoCHIV). Determinants of Vaccine Immunity in the Cohort of Human Immunodeficiency Virus-Infected Children Living in Switzerland. Pediatr Infect Dis J. 2009; 28:996-1001. [PubMed: 19820427]

8. Zinna SS, Bamford A, Cunnington A, et al. Immunization status of children with HIV: failure to protect a vulnerable population. HIV Med. 2011; 12:447-448. [PubMed: 21729230]

9. Schulte JM, Burkham S, Squires JE, et al. Immunization status of children born to human immunodeficiency virus (HIV)-infected mothers in two Texas cities. South Med J. 2000; 93:48-52. [PubMed: 10653065]

10. Ndirangu J, Bärnighausen T, Tanser F, et al. Levels of childhood vaccination coverage and the impact of maternal HIV status on child vaccination status in rural KwaZulu-Natal, South Africa. Trop Med Int Health. 2009; 14(11):1383-1393. [PubMed: 19737375]

11. Hazra R, Stoszek SK, Freimanis-Hance L, et al. NISDI Pediatric Study Group 2008. Cohort Profile: NICHD International Site Development Initiative (NISDI): a prospective, observational study of HIV-exposed and HIV-infected children at clinical sites in Latin American and Caribbean countries. Int J Epidemiol. 2009; 38:1207-1214. [PubMed: 19036797]

12. Burton A, Monasch R, Lautenbach B, et al. WHO and UNICEF estimates of national infant immunization coverage: methods and processes. Bull World Health Organ. 2009; 87(7):535-541. [PubMed: 19649368]

13. De Moraes, JC.; Luna, EA.; Barbosa, H., et al. Research on vaccine coverage in urban areas in capital cities. Brazil: 2007. Available at: http://portal.saude.gov.br/portal/arquivos/pdf/ICV.pdf

14. Ciaranello AL, Chang Y, Margulis AV, et al. Effectiveness of pediatric antiretroviral therapy in resource-limited settings: a systematic review and meta-analysis. Clin Infect Dis. 2009; 49:19151927. [PubMed: 19916798]

15. Moss WJ, Clements CJ, Halsey NA. Immunization of children at risk of infection with human immunodeficiency virus. Bull World Health Organ. 2003; 81:61-70. [PubMed: 12640478]

16. Hesseling AC, Johnson LF, Jaspan H, et al. Disseminated bacille Calmette-Guérin disease in HIVinfected South African infants. Bull World Health Organ. 2009; 87(7):505-511. [PubMed: 19649364]

17. Gamboa Cardeña JR, Morales Pérez DM, Pavía Ruz N, Villalobos Acosta CP. Secondary complications to BCG vaccine in children with HAART. Enf Inf Microbiol. 2001; 31(1):11-16.

18. Campos-Fernandez CS, Araújo LC, Medina-Acosta E. Reduced rate of adverse reactions to the BCG vaccine in children exposed to the vertical transmission of HIV infection and in HIVinfected children from an endemic setting in Brazil. Eur J Pediatr. 2009; 168:691-696. [PubMed: 18766374]

19. Ion-Nedelcu N, Dobrescu A, Strebel PM, et al. Vaccine associated paralytic poliomyelitis and HIV infection. Lancet. 1994; 343:51-52. [PubMed: 7905058] 
20. Chitsike I, van Furth R. Paralytic poliomyelitis associated with live oral poliomyelitis vaccine in child with HIV infection in Zimbabwe: case report. BMJ. 1999; 318:841-843. [PubMed: 10092261]

21. Abzug MJ, Qin M, Levin MJ, et al. for the International Maternal Pediatric Adolescent AIDS Clinical Trials Group P1024 P1061s Protocol Teams. Immunogenicity, Immunologic Memory, and Safety Following Measles Re-Vaccination in HIV-Infected Children Receiving Highly Active Antiretroviral Therapy. J Infect Dis. 2012 Jun 12. Epub ahead of print.

22. Sutcliffe CG, Moss WJ. Do children infected with HIV receiving HAART need to be revaccinated? Lancet Infect Dis. 2010; 10(9):630-642. [PubMed: 20797645]

23. Geretti AM, Doyle T. Immunization for HIV-positive individuals. Curr Opin Infect Dis. 2010; 23:32-38. [PubMed: 19949327] 
Table 1

Demographics of children enrolled in NISDI born from 2002-2007 by HIV status

\begin{tabular}{|c|c|c|c|c|}
\hline \multirow[b]{2}{*}{ Variable name } & \multirow[b]{2}{*}{$\begin{array}{l}\text { Total } \\
1156\end{array}$} & \multicolumn{2}{|c|}{ HIV Status } & \multirow[b]{2}{*}{ P value* } \\
\hline & & $\underset{768(66.4 \%)}{\text { HEU }}$ & $\begin{array}{c}\text { PHIV } \\
388(33.6 \%)\end{array}$ & \\
\hline \multicolumn{5}{|l|}{ Country of birth: } \\
\hline Brazil & 843 & $598(77.9)$ & $245(63.2)$ & $<0.0001$ \\
\hline Mexico & 45 & $1(0.1)$ & $44(11.3)$ & \\
\hline Argentina & 154 & 143 (18.6) & $11(2.8)$ & \\
\hline Peru & 89 & $23(3.0)$ & $66(17.0)$ & \\
\hline Jamaica & 25 & $3(0.4)$ & $22(5.7)$ & \\
\hline \multicolumn{5}{|c|}{ Education of primary caregiver: ${ }^{* *}$} \\
\hline $0-6$ & 398 & $264(34.4)$ & $134(34.9)$ & $<0.0001$ \\
\hline $7-12$ & 662 & $464(60.4)$ & $198(51.6)$ & \\
\hline$\geq 13$ & 92 & $40(5.2)$ & $52(13.5)$ & \\
\hline \multicolumn{5}{|c|}{ Reason for maternal ARV: ${ }^{* *}$} \\
\hline Prophylaxis & 473 & $365(52.1)$ & $108(90.8)$ & $<0.0001$ \\
\hline Treatment & 346 & $335(47.9)$ & $11(9.2)$ & \\
\hline \multicolumn{5}{|c|}{ Tobacco use during pregnancy: } \\
\hline Yes & 252 & $182(23.7)$ & $70(18.0)$ & 0.029 \\
\hline \multicolumn{5}{|c|}{ Alcohol, marijuana, and/or cocaine during pregnancy: } \\
\hline Yes & 122 & $63(8.2)$ & $59(15.2)$ & $<0.0001$ \\
\hline \multicolumn{5}{|l|}{ Gestational age: ${ }^{* *}$} \\
\hline$<37$ weeks & 120 & 84 (12.7) & $36(15.2)$ & 0.319 \\
\hline$\geq 37$ weeks & 780 & $579(87.3)$ & $201(84.8)$ & \\
\hline \multicolumn{5}{|l|}{ Birth weight: } \\
\hline$<2500 \mathrm{~g}$ & 181 & $113(14.8)$ & $68(18.6)$ & 0.118 \\
\hline$\geq 2500 \mathrm{~g}$ & 951 & $653(85.2)$ & $298(81.4)$ & \\
\hline \multicolumn{5}{|l|}{ Sex: } \\
\hline Female & 564 & $371(48.3)$ & $193(49.7)$ & 0.663 \\
\hline \multicolumn{5}{|c|}{ Age of child at enrollment: } \\
\hline$\leq 12$ months & 814 & $768(100)$ & $46(11.9)$ & $<0.0001$ \\
\hline$>12$ to $\leq 24$ months & 82 & 0 & $82(21.1)$ & \\
\hline$>24$ to $\leq 72$ months & 260 & 0 & $260(67.0)$ & \\
\hline
\end{tabular}

P-value obtained from Fisher's exact test.

*** Missing data not included 


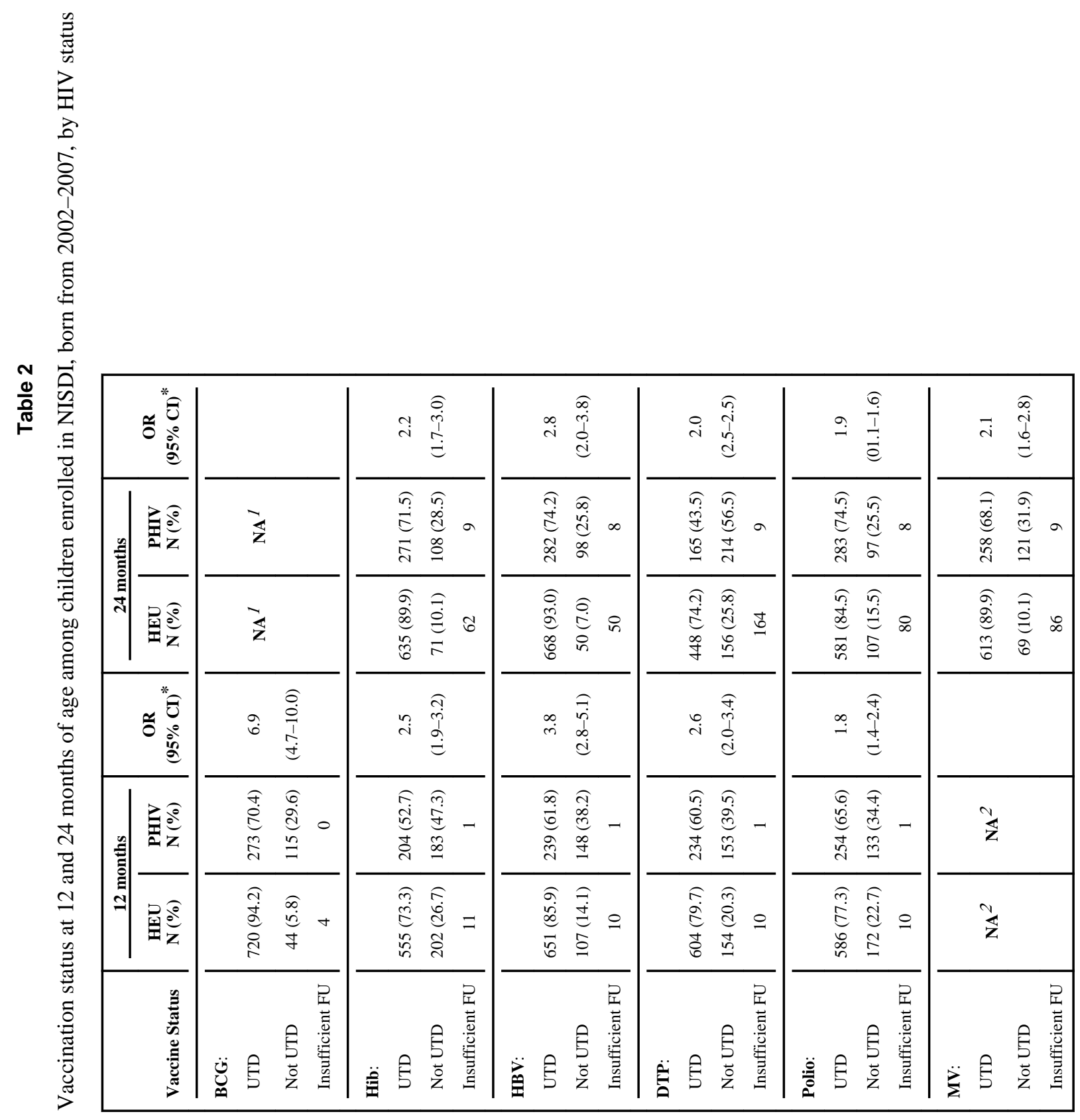

Pediatr Infect Dis J. Author manuscript; available in PMC 2014 August 01. 


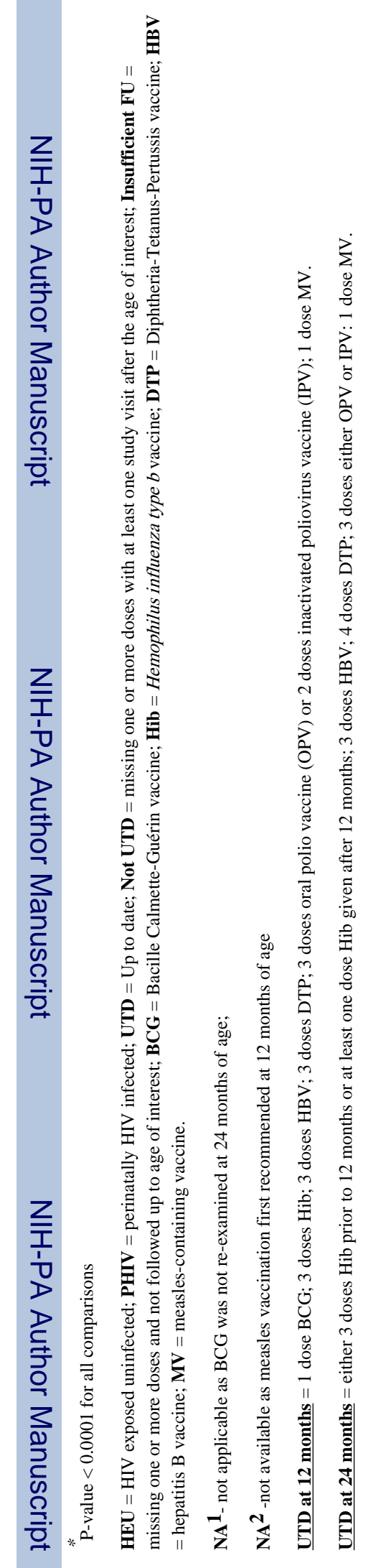

Pediatr Infect Dis J. Author manuscript; available in PMC 2014 August 01. 
Table 3

Vaccination status at 24 months of age among children born from 2002-2007 that were enrolled in NISDI prior to their first birthday, by HIV status

\begin{tabular}{|c|c|c|c|}
\hline Vaccine Status & $\begin{array}{l}\text { HEU } \\
\text { n (\%) }\end{array}$ & $\begin{array}{l}\text { PHIV } \\
\text { n (\%) }\end{array}$ & $\begin{array}{c}\text { Odds Ratio } \\
(\mathbf{9 5 \%} \text { CI })\end{array}$ \\
\hline \multicolumn{4}{|l|}{ Hib: } \\
\hline UTD & $634(89.8)$ & $32(76.2)$ & $2.8(1.3-5.8)$ \\
\hline Not UTD & $72(10.2)$ & $10(23.8)$ & 1.0 \\
\hline Insufficient follow-up & 62 & 4 & \\
\hline \multicolumn{4}{|l|}{ HBV: } \\
\hline UTD & 667 (92.9) & $31(73.8)$ & $4.6(2.2-9.8)$ \\
\hline Not UTD & $51(7.1)$ & $11(26.2)$ & 1.0 \\
\hline Insufficient follow-up & 50 & 4 & \\
\hline \multicolumn{4}{|l|}{ DTP: } \\
\hline UTD & $448(74.2)$ & $21(50.0)$ & $2.9(1.5-5.4)$ \\
\hline Not UTD & $156(25.8)$ & $21(50.0)$ & 1.0 \\
\hline Insufficient follow-up & 164 & 4 & \\
\hline \multicolumn{4}{|l|}{ Polio: } \\
\hline UTD & $581(84.4)$ & $26(61.9)$ & $3.3(1.7-6.4)$ \\
\hline Not UTD & $107(15.6)$ & $16(38.1)$ & 1.0 \\
\hline Insufficient follow-up & 80 & 4 & \\
\hline \multicolumn{4}{|l|}{ MV: } \\
\hline UTD & $613(89.9)$ & $28(66.7)$ & $4.4(2.2,8.8)$ \\
\hline Not UTD & $69(10.1)$ & $14(33.3)$ & 1.0 \\
\hline Insufficient follow-up & 86 & 4 & \\
\hline
\end{tabular}

P-value obtained from Fisher's exact test; unvaccinated children who did not have follow-up to 24 months were not included in deriving p-values.

HEU = HIV exposed uninfected; PHIV = perinatally HIV infected; UTD = Up to date; Not UTD = missing one or more doses with at least one study visit after the age of interest; Insufficient follow-up = missing one or more doses and not followed up to age of interest.

Hib = Hemophilus influenza type $b$ vaccine $\mathbf{H B V}=$ hepatitis B vaccine; $\mathbf{D T P}=$ Diphtheria-Tetanus-Pertussis vaccine $\mathbf{M V}=$ measles-containing vaccine.

$\underline{\text { UTD at } 24 \text { months }}=$ either 3 doses Hib prior to 12 months or at least one dose Hib given after 12 months; 3 doses HBV; 4 doses DTP; 3 doses either OPV or IPV; 1 dose MV. 


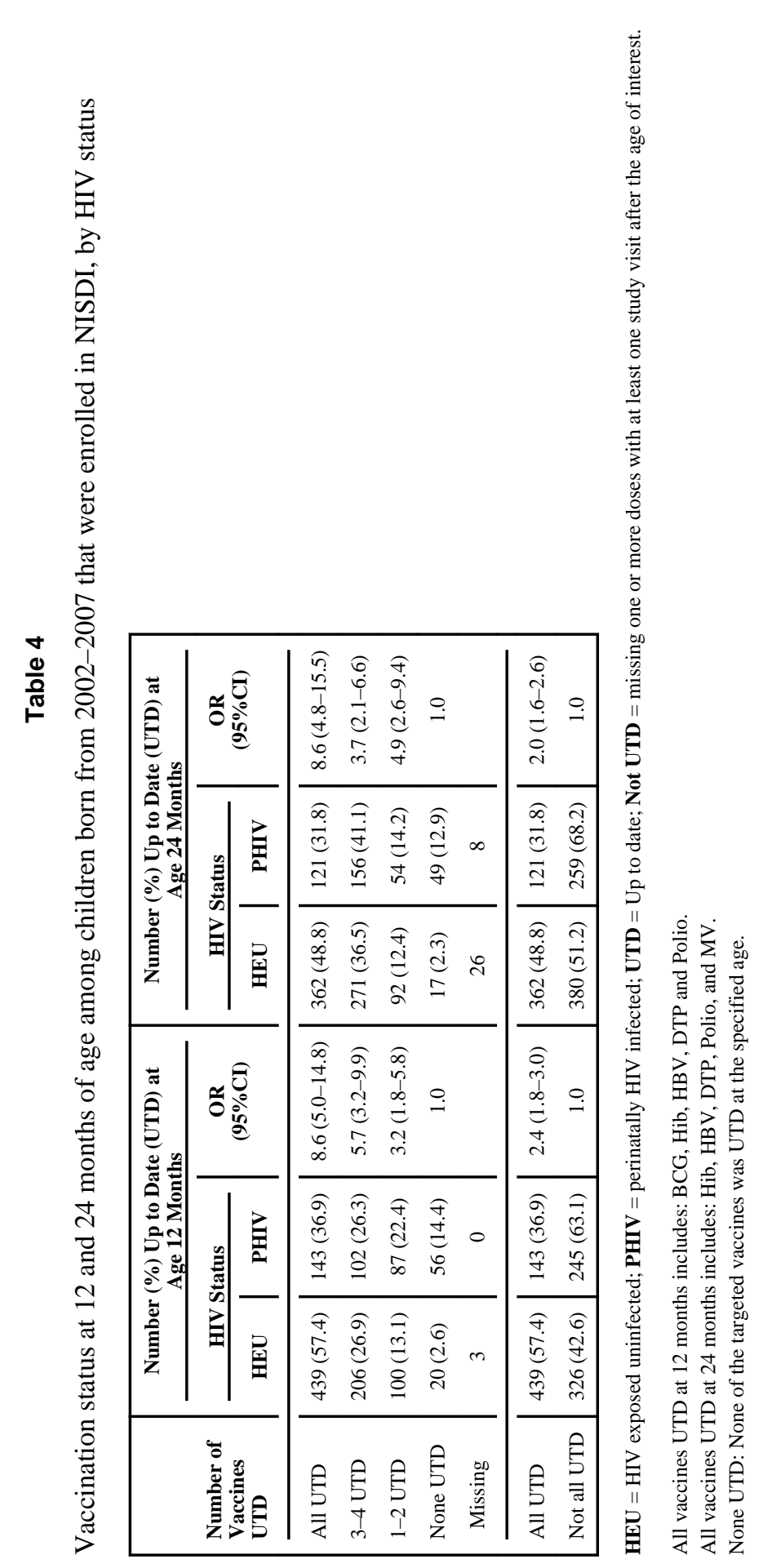

Pediatr Infect Dis J. Author manuscript; available in PMC 2014 August 01. 